\title{
Basophil Activation Test Based on the Expression of CD203C in the Diagnostics of Cow Milk Allergy in Children
}

\author{
O. Ciepiela ${ }^{1}$, J. Zwiazek ${ }^{1}$, A. Zawadzka-Krajewska ${ }^{2}$ I. Kotula1 ${ }^{1}$, M. Kulus ${ }^{2}$, U. Demkow ${ }^{1}$
}

${ }^{1}$ Department of Laboratory Diagnostics and Clinical Immunology of Developmental Age and 2 Department of Pediatric Pneumology and Allergology, Warsaw Medical University, Warsaw, Poland

\begin{abstract}
Background: Detection of allergen-induced basophil activation by flow cytometry has been shown to be a useful tool for allergy diagnosis.

Objective: The aim of this study was to assess the potential of this technique for the diagnosis of pediatric cow milk allergy.

Material and methods: The quantification of total and specific $\operatorname{IgE}$ and basophil activation test were performed to evaluate cow milk allergic $(\mathrm{n}=9)$, and nonallergic children $(\mathrm{n}=15)$.

Results: Allergen-induced basophil activation was detected as a CD203c up-regulation. The expression of CD203c antigen on basophils was measured with flow cytometry. The antigen CD203c was detected on 15.4 $\pm 10.2 \%$ basophils from allergic children after incubation with specific allergens in concentration 1:10, whereas in the control group there were $3.0 \pm 1.5 \%$ of basophils positive for this molecule $(\mathrm{P}<0.05)$. Stimulation with allergen diluted 1:500 resulted in activation of $15.3 \pm 11.2 \%$ of basophils in allergic children and $3.8 \pm 2.3 \%$ of cells in the control group $(\mathrm{P}<0.05)$. Positive results of an allergenicity test (above the cut- off level of $10 \%$ ) were obtained in 7 out of the 9 allergic children. In 5 cases, the cut-off level was reached with both dilution of allergens (1:10 and 1:500). In $1 \mathrm{pa}$ tient, positive stimulation was observed after stimulation with allergen diluted 1:10 and in another case only $1: 500$ resulted in stimulation of more then $10 \%$ of basophils. In no child of the control group, stimulation above $10 \%$ was noted.

Conclusions: This study demonstrates that the analysis of allergen-induced CD203c up-regulation with flow cytometry is a reliable tool for the diagnosis of cow milk allergy in pediatric patients, with sensitivity similar to routine diagnostic tests and a higher specificity.
\end{abstract}

Key words: cow milk allergy, basophil activation test, CD203c, diagnosis, children

\section{INTRODUCTION}

Allergy to cow milk is very common in young children and infants. Digested fractions of the milk proteins may trigger pathological immune response and induce the production of antibodies $[1,2]$. The evaluation of a child suspected of food allergy includes a combina- tion of the several diagnostic tools [1-3]. The diagnosis is made on the basis on medical history and physical examination, prick skin testing, patch testing, in vitro tests, elimination diet, and food challenges. None of the laboratory tests have reached adequate diagnostic accuracy, and the food challenge is connected with a substantial risk of exacerbation of allergy symptoms. Skin prick and patch tests are the most common in vivo tests as the first line investigation. Their positive predictive accuracies, the correlation between the positivity of the test, and the presence of cow milk allergy, vary between 69 and $100 \%$ and the negative predictive values between 20 to $86 \%$ [3]. Prick tests may be negative and the specific $\operatorname{IgE}$ absent especially in children with delayed reactions, a clinical problem which may be overcome by using the atopy patch test (APT) [4]. APT involves prolonged contact of the allergenic food extract with the intact skin under occlusion for 48 h. Positive reactions to patch tests consist of erythema and induration. The measurement of the concentration of food-specific IgE antibodies may be useful when identifying a subset of patients highly likely (>95\% risk) to experience clinical reactions to milk [5].

A high level of milk specific $\mathrm{IgE}$ is useful in diagnosing symptomatic allergy to milk in the pediatric population, but the results remain inconclusive $[1,6$, 7]. In most cases, a placebo-controlled food challenge, a definitive diagnostic test for cow milk allergy, is still required for a subset of patients [1,2]. Newly developed, functional in vitro tests for allergic reactions are focused on circulating basophils and may overcome the need for food challenge tests in some pediatric patients. The first approach to basophil functional studies has been the histamine release test. However, its clinical benefit remains controversial due to insufficient sensitivity and specificity $[8,9]$. The next generation of basophil functional test is based on flow cytometry, as a tool to monitor basophil activation upon allergen challenge by detecting surface expression of activation markers [9]. Basophils and mast cells are the main effector cells responsible for atopic reactions. A large panel of mediators including cytokines, histamine, and tryptase, is released from the cell upon degranulation $[8,9]$. In addition, changes in the expression of basophil surface molecules are observed: some antigens appear de novo (CD63, CD107a) and others significantly increase their expression (CD13, CD203c). The 
CD203c antigen is one of the most important markers of basophil activation. This antigen belongs to the type II transmembrane protein family and is a multifunctional ecto-enzyme called ectonucleotide pyrophosphatase phophodiesterase 3 (E-NPP3) $[9,10]$ that catalyzes the cleavage of a number of molecules including deoxynucleotides and nucleotide sugars. In addition, ENPP3 contains a somatomedin B-like domain and a cell adhesive motif, but their potential functions remain unknown.

Among leukocytes, CD203c appears to be selectively expressed in the basophil/mastocytes lineage [9]. Its expression increases after basophil degranulation and, therefore, is a reliable marker of basophil activation in the IgE-dependent process. Resting basophils are characterized by a low expression of this protein which rapidly increases upon activation [9]. Another molecule that can facilitate basophil recognition is CRTH2 (chemoattractant receptor-homologous molecule expressed on Th2 cells) as it is highly expressed on basophils. CRTH2 is present only on cells associated with Th2 responses (Th2 lymphocytes, eosinophils and basophils). Consequently, a new threecolor flow cytometric protocol (PE-CD203c / FITCCRTH2 / PC5-CD3) for the assessment of allergeninduced basophil activation has been developed [11, 12]. CRTH2 and CD203c staining allows for an easy distinguishing of basophils from other cells in samples of whole blood.

The goal of this study was to assess the usefulness of a basophil activation test, based on the CD203c expression, in the diagnosis of cow milk allergy in children.

\section{Material ANd Methods}

The experiments were approved by the Ethics Committee of Warsaw Medical University in Warsaw, Poland. The blood for the analysis in this study was collected with parental approval. Nine infants and children aged 5-35 months (median 11 months), suffering from cow milk allergy, confirmed with in vitro specific $\mathrm{IgE}$ test positive to milk allergens (Unicap, Pharmacia, Uppsala, Sweden), served as the study group. The blood had been drawn before milk-free diet was introduced. All allergic children showed features of eczema or gastrointestinal symptoms after ingestion of cow milk. 15 healthy, non-allergic controls aged 5-95 months (median 26 months) were included in the control group. None of the subjects were treated with antihistamine drugs or oral corticosteroids.

\section{Basophil Activation Tests}

All tests were carried out within two hours from blood collection. A residual blood sample of $400 \mu$, remaining from routine blood count analysis was used for the basophil activation test. CD203c induced expression was evaluated using the Allergenicity Kit (Beckman Coulter, U.K.) according to the manufacturer's instructions. Milk proteins suspension for prick tests (Allergopharma, Reinbek, Germany) was diluted $1: 10$ and 1:500 in phosphate buffered saline (PBS). EDTA-anticoagulated peripheral blood aliquots (100 $\mu \mathrm{l})$ stained with $20 \mu \mathrm{l}$ of mixture of monoclonal antibodies (CRTH2-FITC, CD203c-PE, CD3-PC7) and Activation Solution $(100 \mu \mathrm{l})$ were stimulated $\left(37^{\circ} \mathrm{C}\right)$ for 15 min with $20 \mu \mathrm{l}$ of optimal dilution of allergens; antibody directed against the high affinity $\mathrm{IgE}$ receptor (FcERI) (Beckman Coulter, U.K.) was used as a positive control and PBS as a negative control. After incubation, the reaction was stopped with Stop Solution. Erythrocytes were lysed with Lysing Solution for $10 \mathrm{~min}$ at room temperature (RT). Suspension was centrifuged ( $5 \mathrm{~min}, 300 \mathrm{x}$ ) after lysing, washed with PBS, once more centrifuged and resuspended in 500 $\mu l$ Fixative Solution. Leukocytes were analyzed using a five-color flow cytometer (Cytomics FC500, Beckman Coulter, U.K.). During acquisition, basophils were selected as CD203c positive/CRTH2 high/CD3 negative population using FL1/FL2 and SS/FL5 dot plots. In the negative control, the threshold for positivity was set at less than $5 \%$ of activated cells according to the literature data $[1,9]$. In the positive control, sensitivity for the $\operatorname{IgE}$ dependent reaction was verified. The threshold for a positive reaction was settled at less than $15 \%$ of activated cells, according to literature data and the investigators' previous studies [11, $12]$.

\section{StATISTICAL ANALYSIS}

Results are presented as means $\pm \mathrm{SD}$. A comparison of the results from different groups was performed using Mann-Whitney $\mathrm{U}$ test. $\mathrm{P}<0.05$ was considered significant. Specificity and sensitivity were calculated according to following equations:

sensitivity $=\mathrm{TP} /(\mathrm{TP}+\mathrm{FN}) \times 100 \%$ and specificity $=$ $\mathrm{TN} /(\mathrm{TN}+\mathrm{FP})$ x 100\%; where: TP - true positive, FD false positive, TN - true negative, $\mathrm{FN}$ - false negative results.

\section{RESULTS}

The CD203c expression on basophils was evaluated to assess patients' sensitivity to cow milk antigen stimulation. The mean fluorescence of fluorochrome bound to CD203c was measured before and after allergenic stimulation. As a negative control, fluorescence of spontaneously activated basophils was measured. As a positive control, fluorescence of $\mathrm{IgE}$ stimulated basophil was estimated (Fig. 1). The cut-off level was set at $>10 \%$ CD 203 c positive cells.

The mean percentages of activated basophils in the allergic children were $3.8 \pm 0.7 \%$ (negative control) and $22.2 \pm 13.9 \%$ (positive control). Accordingly, in the group of control children, the mean percentage of cells with CD203c expression was $3.6 \pm 1.2 \%$ (negative control) and $31.7 \pm 16.5 \%$ (positive control). The mean fluorescence of non-stimulated cells in the allergic children was $13.3 \pm 7.3 \mathrm{MFC}$ and that in the control children was $17.4 \pm 9.7 \mathrm{MFC}$; the difference being insignificant. The presence of CD203c was detected on 15.4 $\pm 10.2 \%$ basophils from allergic children after incubation with specific allergens in the concentration 1:10, whereas in the control group $3.0 \pm 1.5 \%$ of basophils were positive for this molecule $(\mathrm{P}<0.05)$. Stimulation with the allergen diluted 1:500 resulted in the activation 

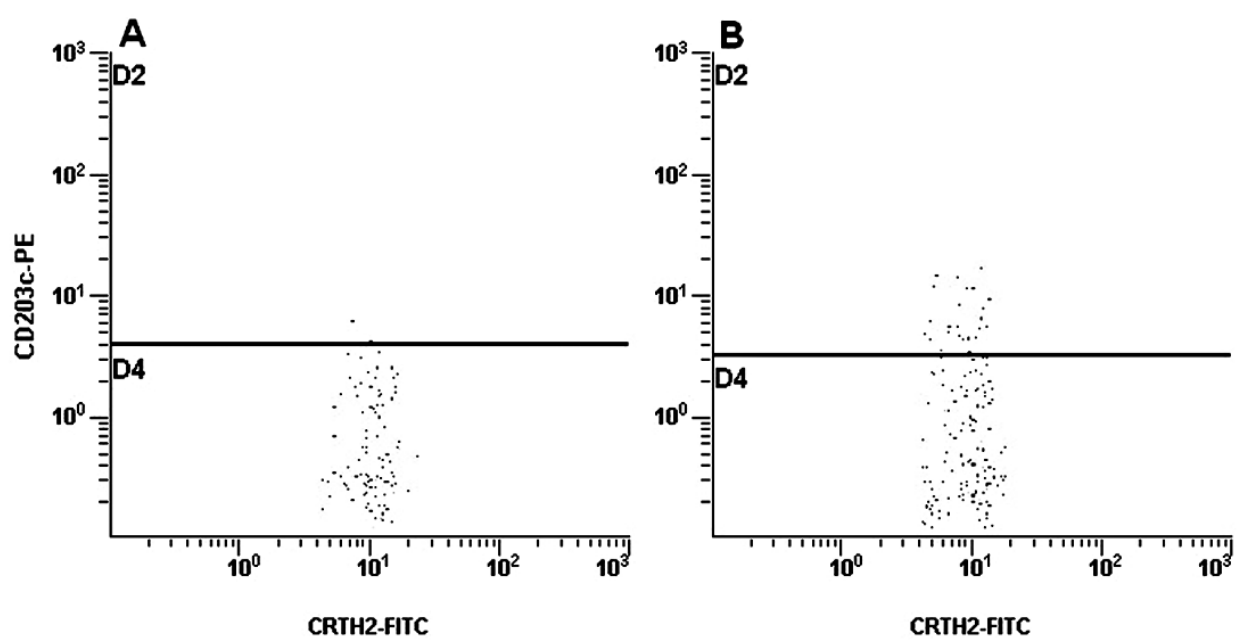

Fig. 1. Expression of CD203c in negative (A) and positive (B) controls.

Table 1. Percentage of activated basophils in the group of cow milk allergic children.

\begin{tabular}{|c|c|c|c|c|c|}
\hline No & $\begin{array}{l}\text { Activated cells in } \\
\text { cells in } \\
\text { negative } \\
\text { control }(\%)\end{array}$ & $\begin{array}{l}\text { Activated } \\
\text { cells in } \\
\text { positive } \\
\text { control }(\%)\end{array}$ & $\begin{array}{l}\text { Activated cells } \\
\text { after stimulation } \\
\text { with allergens } \\
\text { diluted 1:10 }(\%)\end{array}$ & $\begin{array}{l}\text { Activated cells after } \\
\text { stimulation with } \\
\text { allergens diluted } \\
1: 500(\%)\end{array}$ & $\begin{array}{c}\text { Fluorescence of } \\
\text { spontaneously } \\
\text { activated basophils } \\
\text { (MFC units) }\end{array}$ \\
\hline 1 & 2.8 & 51.4 & 1.7 & 5.3 & 4.9 \\
\hline 2 & 3.5 & 29.9 & 33.1 & 37.3 & 6.3 \\
\hline 3 & 4.1 & 15.4 & 16.9 & 3.7 & 13.3 \\
\hline 4 & 3.1 & 5.0 & 9.3 & 4.6 & 11.0 \\
\hline 5 & 4.7 & 16.2 & 15.6 & 16.7 & 18.7 \\
\hline 6 & 4.5 & 17.5 & 23.1 & 26.2 & 25.2 \\
\hline 7 & 4.1 & 33.3 & 5.9 & 11.8 & 11.7 \\
\hline 8 & 3.9 & 18.2 & 13.1 & 19.9 & 6.4 \\
\hline 9 & 3.5 & 13.1 & 21.2 & 11.8 & 22.1 \\
\hline
\end{tabular}

The results above the cut-off level are bolded.

Table 2. Percentages of activated basophils in the control group.

\begin{tabular}{lccccc}
\hline No & $\begin{array}{c}\text { Activated cells in } \\
\text { cells in } \\
\text { negative } \\
\text { control }(\%)\end{array}$ & $\begin{array}{c}\text { Activated } \\
\text { cells in } \\
\text { positive } \\
\text { control }(\%)\end{array}$ & $\begin{array}{c}\text { Activated cells } \\
\text { after stimulation } \\
\text { with allergens } \\
\text { diluted 1:10 (\%) }\end{array}$ & $\begin{array}{c}\text { Activated cells after } \\
\text { stimulation with } \\
\text { allergens diluted } \\
1: 500(\%)\end{array}$ & $\begin{array}{c}\text { Fluorescence of } \\
\text { spontaneously } \\
\text { activated basophils } \\
\text { (MFC units) }\end{array}$ \\
\hline 1 & 4.7 & 67.3 & 1.9 & 3.1 & 16.0 \\
2 & 1.9 & 44.2 & 3.3 & 7.3 & 7.6 \\
3 & 4.3 & 20.2 & 2.8 & 0.8 & 22.0 \\
4 & 4.7 & 23.8 & 2.7 & 7.5 & 20.0 \\
5 & 2.6 & 20.7 & 0.6 & 1.0 & 7.0 \\
6 & 3.9 & 16.1 & 3.6 & 5.0 & 18.9 \\
7 & 1.2 & 15.4 & 1.9 & 1.6 & 11.8 \\
8 & 2.4 & 11.8 & 0.0 & 0.0 & 18.5 \\
9 & 3.3 & 16.2 & 5.6 & 5.2 & 34.5 \\
10 & 4.7 & 42.3 & 4.1 & 3.8 & 32.5 \\
11 & 3.3 & 29.7 & 2.3 & 3.1 & 6.8 \\
12 & 5.4 & 29.0 & 4.4 & 5.7 & 32.7 \\
13 & 3.8 & 52.7 & 4.1 & 4.1 & 14.5 \\
14 & 4.6 & 37.0 & 4.6 & 3.8 & 5.9 \\
15 & 3.5 & 49.3 & 3.9 & 4.6 & 12.1 \\
\hline
\end{tabular}


Table 3. Percentage of Th2 cells among lymphocytes (\%).

\begin{tabular}{lcc}
\hline No & $\begin{array}{c}\text { Allergic children } \\
\text { children }\end{array}$ & $\begin{array}{c}\text { Healthy children } \\
\text { children }\end{array}$ \\
\hline 1 & 0.2 & 0.4 \\
2 & 0.3 & 0.3 \\
3 & 0.4 & 0.4 \\
4 & 0.4 & 0.6 \\
5 & 0.3 & 0.1 \\
6 & 0.3 & 0.4 \\
7 & 0.1 & 0.0 \\
8 & 2.3 & 0.3 \\
9 & 2.8 & 1.2 \\
10 & -- & 0.7 \\
11 & -- & 0.5 \\
12 & -- & 0.6 \\
13 & -- & 2.8 \\
14 & -- & 2.6 \\
15 & -- & 0.8 \\
\hline
\end{tabular}

Table 4. True positive (TP), false positive (FP), true negative (TN), and false negative (FN) results.

\begin{tabular}{ccc}
\hline $\begin{array}{c}\text { Basophil } \\
\text { activation test }\end{array}$ & \multicolumn{2}{c}{$\begin{array}{c}\text { Presence of cow milk allergy } \\
\text { Yes }\end{array}$} \\
\hline Positive & TP $(\mathrm{n}=12)$ & $\mathrm{FP}(\mathrm{n}=0)$ \\
Negative & $\mathrm{FN}(\mathrm{n}=6)$ & $\mathrm{TN}(\mathrm{n}=30)$ \\
Together & $\mathrm{TP}+\mathrm{FN}(\mathrm{n}=18)$ & $\mathrm{FP}+\mathrm{TN}(\mathrm{n}=30)$ \\
\hline
\end{tabular}

of $15.3 \pm 11.2 \%$ of basophils in the allergic children and $3.8 \pm 2.3 \%$ of cells in the control group $(\mathrm{P}<0.05)$.

Positive results of the allergenicity test were obtained in 7 out of the 9 allergic children. In 5 cases, the cut-off level was reached with both dilutions of allergens (1:10 and 1:500). In 1 patient, positive stimulation was observed after stimulation with the allergen diluted 1:10 and in another case only 1:500 resulted in stimulation of more than $10 \%$ of basophils. In 2 patients, significant basophil activation was not observed. In no child of the control group was the stimulation above $10 \%$ noted. In 4 healthy children, the percentage of activated basophils was $5-10 \%$. The individual results are summarized in Tables 1 and 2 .

The percentage of Th2 cells among lymphocytes in both allergic and non-allergic children was calculated. In the children allergic to cow milk, the percentage of Th2 lymphocytes was $0.12-2.8 \%$ (median 0.33 ) and that in the control group was $0.02-2.8 \%$ (median 0.48); the difference being insignificant. These results are presented in Table 3. Diagnostic accuracy of the basophil activation test is presented in Table 4. The test sensitivity was $66.7 \%$ and the specificity was $100 \%$.

\section{DisCUSSION}

Basophil activation with specific allergens has proven to be useful for diagnosis of pollen, latex, and food allergy [13]. CD203c up-regulation is more or less specific to the cross linking of FceRI. Hence, as CD203c is rapidly up-regulated after allergen challenge, it has been proposed as a new tool for allergy diagnosis [9, 13-15]. Our study demonstrates that the analysis of allergen-induced CD203c up-regulation by flow cytometry is a reliable tool for diagnosis of cow milk allergy in pediatric patients, with sensitivity similar to routine diagnostic tests and a higher specificity than that of specific $\mathrm{IgE}$ determination. Therefore, the basophil activation test is a highly efficient in confirming cow milk allergy in children. Several authors have studied the diagnostic value of basophil activation test in food allergy. Ocmant et al [16] have found sensitivity of $89.5 \%$ and specificity of $97.1 \%$ in peanut allergy and that of $62.5 \%$ and $96.4 \%$, respectively, in egg allergy. Tokuda et al [17] have confirmed $85.0 \%$ sensitivity and $77.0 \%$ specificity in wheat allergy. In the present study, two of the examined patients with confirmed cow milk allergy presented a negative result of the basophil activation test. At least in one case, this result may be explained by a relatively low basophil count in the peripheral blood. In this patient, the response to positive control also was low (4.95\%). The cause of the unresponsiveness in another case is not known. As $40-50 \%$ of milk allergy is non- $\mathrm{IgE}$ mediated [18], we may speculate that in this patient the allergy was induced by IgE-unrelated mechanisms. Two of the examined children responded to activation with only one solution of the allergen. It confirms the opinion of others that different concentrations of allergen may stimulate or block basophil response [19-21]. Interestingly, two of the control children also responded in a concentration-dependent manner; however, in neither case the obtained result exceeded the cut-off value. We also observed in a child from the control group that anti-IgE stimulation (positive control) did not result in increased expression of CD203c on basophils. This may be explained by disturbances in intracellular signaling commonly observed in around $5 \%$ of the general population (non-responders) [11]. On the other hand, the identification of basophils using prior protocols relied on a single IgE-labeling, showed that FceRI expression can vary considerably on cell surfaces from one patient to another [22]. That is why in some cases basophils were difficult or impossible to be stimulated by anti$\mathrm{IgE}$ monoclonal antibodies.

In the present study we also showed that there was no difference between the Th2 populations of cells in the allergic and non-allergic children. The lack of difference may be explained by the immaturity of the immune system in young children. Moreover, CRTH2staining protocol, illustrated its superiority with respect to basophil recovery. Easy recognition of basophils and a reliable assessment of their activation make this protocol the most reliable tool for investigating basophil activation by flow cytometry. Since CRTH2 is also a marker of Th2 cells and eosinophils, it may become a promising tool for flow cytometry, providing a direct overview of cells involved in 'Th2 diseases', such as allergy $[12,16,17]$.

It is a common observation that food allergy is a disease of young children, which in most cases is out- 
grown. The majority of children develop tolerance within the first 3-5 years of life [23]. The two foods responsible for most of these reactions are cow milk and hen eggs. It is then obvious that a reliable test to predict loss of sensitivity is needed. Children who acquire tolerance can continue to have positive skin test results for years, and therefore skin tests are not predictive of outgrowing the food sensitivity $[23,24]$. A positive double-blind, placebo-controlled food challenge is the gold standard for the diagnosis of food allergy $[1,2]$. After initiating a cow milk restriction diet, the clinical manifestations triggered by the first challenge are difficult to predict. In contrast, if the first challenge fails, further ones may result in severe symptoms. One must also be prepared to conduct challenges in a safe environment with available facilities for resuscitation. Rubio et al [25] have shown the benefit of a basophil activation test in predicting the child's reaction to the oral challenge, evaluating and comparing the specific $\operatorname{IgE}$ and skin prick test results. The authors used allergen-induced basophil activation based on CD63-upregulation by flow cytometry. The test had efficiency of $90 \%$, sensitivity of $91 \%$, and specificity of $90 \%$, and positive and negative predictive values were $81 \%$ and $96 \%$, respectively, in detecting persistently allergic patients [25]. According to this observation, a basophil activation test may be a useful and safe tool in predicting outgrowing the food sensitivity in children. To date, there are no good indices to predict when and in whom this occurs.

We conclude that a basophil activation test presents with high specificity and relatively low sensitivity in cow milk allergy in children. Basophil activation depends on the concentration of the allergen. As flow cytometry is a valuable tool for the analysis of many different cell types and can be used to identify specific populations of cells, even when present in low numbers, it seemed to be suitable for a study of allergeninduced basophil degranulation. Th2 analysis is not a good parameter discriminating allergic and non-allergic children.

Conflicts of interest: The authors declared no conflict of interest in relation to this article.

\section{REFERENCES}

1. de Boissieu D, Dupont C. Time course of allergy to extensively hydrolyzed cow milk proteins in infants. J Pediatr 2000; 136: 119-20.

2. de Boissieu D, Dupont C. Allergy to extensively hydrolyzed cow milk proteins in infants: safety and duration of amino acid-based formula. J Pediatr 2002; 41: 271-3.

3. Fiocchi A, Bouygue GR, Restani P, Bonvini G, Startari R, Terracciano L. Accuracy of skin prick tests in IgE-mediated adverse reactions to bovine proteins. Ann Allergy Asthma Immunol 2002; 89 (6 Suppl 1): 26-32.

4. de Boissieu D, Waguet JC, Dupont C. The atopy patch tests for detection of cow milk allergy with digestive symptoms. J Pediatr 2003; 142: 203-5.

5. Sampson HA, Ho DG. Relationship between food-specific $\mathrm{IgE}$ concentrations and the risk of positive food challenges in children and adolescents. J Allergy Clin Immunol 1997; 100: 444-51.
6. Isolauri E, Turjanmaa K. Combined skin prick and patch testing enhances identification of food allergy in infants with atopic dermatitis. J Allergy Clin Immunol 1996; 97: 9-15.

7. Heine RG, Elsayed S, Hosking CS, Hill DJ. Cow milk allergy in infancy. Curr Opin Allergy Clin Immunol 2002; 2: 217-25.

8. Hamilton RG, Adkinson NF. In vitro assays for the diagnosis of IgE-mediated disorders. J Allergy Clin Immunol 2004; 114: 213-25.

9. Boumiza R, Debard AL, Monneret G. The basophil activation test by flow cytometry: recent developments in clinical studies, standardization and emerging perspectives Clin Mol Allergy 2005; 3: 9-13.

10. Buhring HJ, Seiffert M, Giesert C, Marxer A, Kanz L, Valent $\mathrm{P}$, Sano K. The basophil activation marker defined by antibody $97 \mathrm{~A} 6$ is identical to the ectonucleotide pyrophosphatase/phosphodiesterase 3. Blood 2001; 97: 3303-5.

11. Potapinska O, Gorska E, Zawadzka-Krajewska A, Kulus M, Wasik M, Demkow U. The usefulness of CD203c expression measurement on basophils after activation with grass pollen and Dermatophagoides pteronyssinus antigens. Preliminary study. Pneumonol Alergol Pol 2009; 77: 138-44.

12. Potapinska O, Demkow U, Wasik M. Flow cytometric basophils activation test as a method of allergy diagnosis. Pneumonol Alergol Pol 2009; 77: 152-8.

13. Gonzalez-Muñoz M, Villota J, Moneo I. Analysis of basophil activation by flow cytometry in pediatric house dust mite allergy. Pediatr Allergy Immunol 2002; 19: 3427.

14. Bühring HJ, Streble A, Valent P. The basophil-specific ectoenzyme E-NPP3 (CD203c) as a marker for cell activation and allergy diagnosis. Int Arch Allergy Immunol 2004; 133: 317-29.

15. Kahlert H, Cromwell O, Fiebig H. Measurement of basophil-activating capacity of grass pollen allergens, allergoids and hypoallergenic recombinant derivatives by flow cytometry using anti-CD203c. Clin Exp Allergy 2003; 33: 1266-72.

16. Ocmant A, Mulier S, Hanssens L, Goldman M, Casimir G, Mascart F. Basophil activation tests for the diagnosis of food allergy in children. Clin Exp Allergy 2009; 39: 1234-45.

17. Tokuda R, Nagao M, Hiraguchi Y, Hosoki K, Matsuda T, Kouno K, Morita E, Fujisawa T. Antigen-induced expression of CD203c on basophils predicts IgE-mediated wheat allergy. Allergol Int 2009; 58: 193-9.

18. Host A, Halken S. A prospective study of cow milk allergy in Danish infants during the first 3 years of life. Clinical course in relation to clinical and immunological type of hypersensitivity reaction. Allergy 1990; 45: 587 96.

19. Boumiza R, Debard A, Monneret G. The basophil activation test by flow cytometry: recent developments in clinical studies, standardization and emerging perspectives. Clin Mol Allergy 2005; 3: 9-14.

20. Michova A, Abugalia M, Ivanova T, Nikolov G, Taskov H, Petrunov B. Comparison of two flow cytometry methods for basophil degranulation in patients sensitized to grass pollen. Allergy 2006; 61: 1078-83.

21. Monneret G, Gutowski M, Bienvenu J. Detection of allergen-induced basophil activation by expression of CD63 antigen using a tricolour flow cytometric method. Clin Exp Allergy 1999; 115: 393-6.

22. Kinet JP. The high-affinity IgE receptor (FcદRI): From physiology to pathology. Ann Rev Immunol 1999; 17: 931-72.

23. Bock SA. The natural history of food sensitivity. J Allergy Clin Immunol 1982; 69: 173-7. 
24. Sampson HA, Scanlon SM. Natural history of food hypersensitivity in children with atopic dermatitis. J Pediatr 1989; 115: 23-7.

25. Rubio A, Vivinus-Nébot M, Bourrier T, Saggio B, Albertini M, Bernard A. Benefit of the basophil activation test in deciding when to reintroduce cow milk in allergic children. Allergy 2010; DOI: 10.1111/j.1398-9995.2010.02432.
Address for correspondence:

Olga Ciepiela

Department of Laboratory Diagnostics and

Clinical Immunology of Developmental Age

Warsaw Medical University

24 Marszalkowska St.

00-576 Warsaw

Poland

E-mail: olga.ciepiela@wum.edu.pl 